\title{
Neurophotonics
}

\section{Infrared neural stimulation: a new stimulation tool for central nervous system applications}

Mykyta Chernov

Anna Wang Roe 


\title{
Infrared neural stimulation: a new stimulation tool for central nervous system applications
}

\author{
Mykyta Chernov and Anna Wang Roe* \\ Vanderbilt University, Department of Psychology, 111 21st Avenue South, Nashville, Tennessee 37240, United States
}

\begin{abstract}
The traditional approach to modulating brain function (in both clinical and basic science applications) is to tap into the neural circuitry using electrical currents applied via implanted electrodes. However, it suffers from a number of problems, including the risk of tissue trauma, poor spatial specificity, and the inability to selectively stimulate neuronal subtypes. About a decade ago, optical alternatives to electrical stimulation started to emerge in order to address the shortcomings of electrical stimulation. We describe the use of one optical stimulation technique, infrared neural stimulation (INS), during which short (of the order of a millisecond) pulses of infrared light are delivered to the neural tissue. Very focal stimulation is achieved via a thermal mechanism and stimulation location can be quickly adjusted by redirecting the light. After describing some of the work done in the peripheral nervous system, we focus on the use of INS in the central nervous system to investigate functional connectivity in the visual and somatosensory areas, target specific functional domains, and influence behavior of an awake nonhuman primate. We conclude with a positive outlook for INS as a tool for safe and precise targeted brain stimulation. $\odot$ The Authors. Published by SPIE under a Creative Commons Attribution 3.0 Unported License. Distribution or reproduction of this work in whole or in part requires full attribution of the original publication, including its DOI. [DOI: 10.1117/1.NPh.1.1.011011]
\end{abstract}

Keywords: infrared neural stimulation; optical imaging; nonhuman primate; cerebral cortex.

Paper 14027VSSRR received Mar. 12, 2014; revised manuscript received Jun. 26, 2014; accepted for publication Jul. 1, 2014; published online Aug. 5, 2014.

\section{Introduction}

Scientific literature is rife with analogies between the brain and a computer microchip. This electricity-centered view generated a lot of optimism about the development of brain-machine interfaces, which would take over diseased brain function or somehow enhance the brain's natural capacities using vast arrays of electrical wires. Once only science fiction, over the past few decades such a vision has indeed become a reality with the advent of high-density electrode arrays. However, progress has been impeded by several obstacles which are unlikely to be resolved quickly. First, the brain, unlike most electronic circuits, has a three-dimensional architecture, and any attempt to tap into the deeper layers requires insertion of foreign objects into living tissue. These implants can only be so dense before they start causing trauma, setting a limit on their resolving power. ${ }^{1}$ Second, electrode arrays are unable to target specific neuronal types located with the same region. Third, the magnitude of the electrical stimulus and its spatial extent are not independent factors - the higher the stimulus applied at the site of interest, the greater is the chance of undesired activation of the surrounding tissue.

About 10 years ago, scientists started developing optical alternatives to electrical stimulation, attempting to access neurons using beams of light rather than wires. Karl Diesseroth, Edward Boyden, and their colleagues at Stanford and MIT pioneered the field of optogenetics. ${ }^{2}$ Not only does their method eliminate the need for wires, but also enables targeting of neuronal subtypes by tying the expression of the optical switches to specific promoters. It also allows for inhibition as well as activation of channels, as well as for a bistable mode of operation

*Address all correspondence to: Anna Wang Roe, E-mail: Anna.Roe@ Vanderbilt.edu where the optical element is turned on by one wavelength and off by another. ${ }^{3}$ Optogenetics has been successfully applied in rodents, but has not been as useful in nonhuman primates, and safety concerns still bar it from use in humans.

Another optical stimulation technique, infrared neural stimulation (INS), was developed at approximately the same time as optogenetics by Duco Jansen and colleagues at Vanderbilt University. ${ }^{4}$ It employs brief (of the order of $1 \mathrm{~ms}$ ) pulses of infrared light to depolarize the neuronal membrane and generate action potentials. The mechanism of INS is due to transient and localized heating caused by absorption of infrared light by water. ${ }^{5}$ While optogenetics relies on introducing algal optical switches into mammalian tissue, INS works at a more basic level and does not require genetic manipulation. ${ }^{6}$ Its advantages over electrical stimulation include very high spatial precision, with stimulation essentially confined to the illumination spot, which is typically 100 to 400 microns in diameter. Stimulation of superficial cortical layers may be done through a transparent membrane without penetration of the tissue itself, and the location of the stimulus may be altered at will by simply redirecting the light beam. ${ }^{7}$ INS has added benefits of not interfering with electrical recordings as long as the electrodes are not within the illumination spot, as well as of being magnetic resonance imaging (MRI) compatible. ${ }^{89}$ In this review, we introduce the reader to INS, describing its mechanism of action, summarizing the early experiments in the peripheral nervous system, and then focusing on its application to the study of cortical function.

\section{Mechanism of INS Action}

Light is a form of electromagnetic energy. When it encounters an absorbing medium, such as neural tissue, it can perturb it in a variety of ways, depending on the wavelength and the shape of the optical pulse both in space and time. ${ }^{10}$ It can break molecular 
bonds, accelerate chemical reactions or even create shock waves that spread through tissue at the speed of sound. All of these optical effects have been shown to influence neuronal activity. ${ }^{5}$ INS was developed with a working hypothesis that rapid and focal changes in temperature can also perturb the transmembrane potential sufficiently to generate action potentials. Given that water is a major constituent of biological tissues, Jansen and coworkers chose it as the absorber of optical energy. Using a free electron laser with a tunable wavelength, they demonstrated that pulses of infrared light induced action potentials in frog and rat sciatic nerves. ${ }^{4}$ The absorption of water increases as a function of wavelength, starting at 1 micron and is punctuated by several peaks, notably at $1.45,1.9$, and 2.9 microns. Jansen et al. found that the optimal wavelength for INS was such that the absorption was high enough to generate the rapid heat transients in the nerve necessary for action potential generation. They further showed that penetration depth of a few hundred microns was found to be ideal (close to the first two absorption peaks and in the valleys at longer wavelengths) and the energy thresholds were $\sim 0.3 \mathrm{~J} / \mathrm{cm}^{2}$, deposited in a pulse ranging from 0.25 to a few milliseconds. ${ }^{8,11}$ [The selection of wavelengths has been limited by availability of light sources of sufficient power, but today some choices include the holmium-YAG laser (2.12 microns), thulium fiber lasers (tunable around 2 microns), and high-power semiconductor lasers (particularly 1450, 1465, and $1875 \mathrm{~nm}$ ). The necessary continuous wave $(\mathrm{CW})$ power ranges from a fraction of a watt to a few watts, depending on the wavelength and experimental setup.] To be effective, INS had to be in the thermal confinement regime and $\mathrm{CW}$ operation was not effective in generating action potentials.

Several ideas, which are not mutually exclusive, have been proposed for the mechanism of transduction of thermal energy to changes in membrane potential. The most basic mechanism is capacitive, proposed by Shapiro et al. ${ }^{6}$ They investigated the changes in transmembrane potentials in reduced preparations using voltage clamp techniques. In Xenopus oocytes, human embryonic kidney cells, and even artificial bilayers, INS depolarized the membrane potential by up to $9 \mathrm{mV}$. If a suitable combination of ion channels was expressed in the oocytes, INS would cause them to fire action potentials, but, importantly, the INS-induced capacitive currents were resistant to various channel blockers. The authors have also shown that the changes in membrane potential can be predicted rather well using the Gouy-Chapman-Stern model of a double capacitor, which ties the transmembrane capacitative charge to temperature and requires INS to be pulsed rather than continuous in order for it to be effective.

Complementary to the capacitative theory of INS action is the hypothesis that temperature changes the conductance of various ion channels. ${ }^{12}$ Most, if not all, ion channels are temperature sensitive to some extent, but the members of the transient receptor potential vanilloid channel (TRPV) family are particularly well known for their thermosensitivity. Albert et al. showed that they were the primary source of sensitivity to INS in explanted retinal and vestibular ganglion neurons, with calcium and sodium voltage-sensitive channels being minor contributors. The presence of TRPV channels in the cochlea has also been proposed as the reason for INS of auditory neurons being effective at radiant exposures one to two orders of magnitude lower than, for example, that in the sciatic nerve of the rat. ${ }^{13}$ One may conclude then that INS has an excitatory effect on the lipid membrane, which may be amplified or even negated by the presence of thermosensitive cellular components, including ion channels and, probably, other, yet unidentified, components as well. Their relative importance is likely to be tissue specific.

\section{Early INS Applications}

INS offers two significant advantages over electrical stimulation: high spatial resolution and contact-free stimulation. The Jansen group suggested that both of these advantages can be exploited in the development of neural prostheses. They demonstrated activation of individual fascicles with INS in the sciatic nerve of frogs and rats and, consequently, control of isolated leg muscles rather than a contraction of the entire limb usually observed when the nerve is stimulated with ring electrodes. ${ }^{4,8}$ Finer electrical control may be achieved by impaling the nerve with microelectrodes, but such preparations often lead to extensive nerve damage. ${ }^{1}$ The high spatial resolution of INS prompted Richter and colleagues to develop an optical cochlear stimulator using a deafened cat model. ${ }^{13}$ INS was found to be superior to electrical stimulation in encoding acoustic frequencies even when only a few INS channels were used. ${ }^{14}$ Finally, the contact-free nature of INS made it attractive for use in delicate structures. Jenkins et al. showed that it can be used for cardiac pacing of embryonic quail hearts as a means of altering their development. ${ }^{15}$

\section{INS of the Central Nervous System}

The cortical surface presents a fundamentally different landscape for optical stimulation in comparison to peripheral nerve stimulation. Rather than being a cable-like arrangement of axons as in peripheral nerves, it is a complex network of excitatory neurons, inhibitory interneurons, various types of glia, as well as vasculature and epithelial tissue. The Jansen group used electrophysiological recordings in combination with intrinsic optical calcium dye and flavoprotein fluorescence imaging in rodents to demonstrate the feasibility of INS application in the cortex, explore the optical parameters necessary for successful stimulation, and determine which cell types are affected by INS. ${ }^{16,17}$

Intrinsic optical signal imaging is a technique used to detect local changes in blood flow via red light reflectance due to focal metabolic changes. ${ }^{18}$ It is a convenient way to detect cortical activation patterns because it requires no contrast agent and can be used to image a relatively wide area (tens of square millimeters) with a resolution of the order of 10 microns. ${ }^{19}$ Cayce et al. showed that INS of the rat somatosensory cortex using a spot size of $\sim 800$ microns produced reflectance changes characteristic of cortical intrinsic signals evoked by sensory tactile stimulation. ${ }^{20}$ Activation was observed at radiant exposures as low as $0.14 \mathrm{~J} / \mathrm{cm}^{2}$ and its size and magnitude (in terms of peak change in red light reflectance) varied linearly as a function of radiant exposure and exponentially as a function of stimulation frequency (which was between 50 and $200 \mathrm{~Hz}$ ). Activation was confined to a region $\sim 1.5 \mathrm{~mm}$ in diameter. The time course of the activation was relatively slow, with the peak of activation occurring $1 \mathrm{~s}$ following the onset of stimulation, which itself lasted for $0.5 \mathrm{~s}$. Such slow dynamics are typical of intrinsic optical imaging. Interestingly, concurrent multiunit electrophysiological recordings showed a decrease in neuronal firing rate following INS. The authors suggest that the seemingly paradoxical finding is related to the large spot size (fiber optic size) used. 
That is, a large spot size could activate the relatively large number of inhibitory interneurons in cortical layers I and II, which normally contribute to inhibitory surrounds of cortical receptive fields and which, when activated, could in turn suppress the neuronal network via synaptic connections.

To further elucidate the origin of the intrinsic optical signal changes following INS, the Jansen group combined calcium and flavin autofluorescence imaging with the use of pharmacological agents in rats. ${ }^{16}$ Using Oregon Green dye as a calcium indicator, they observed wave-like propagation of the calcium signal following INS. The dynamics of the calcium signal consisted of a slow and a fast component. They proposed that the fast component was neuronal in origin, while the slow component was mainly astrocytic. To support their claim, they employed two pharmacological agents, 6-cyano-7-nitroquinoxaline-2,3-dione (CNQX) (which inhibits excitatory synaptic transmission by blocking $\alpha$-Amino-3-hydroxy-5-methyl-4-isoxazolepropionic acid (AMPA) and kainate glutamate receptors) and the astrocytic poison sodium fluoroacetate. Although both drugs inhibited the slow and the fast components of the INS-induced calcium response, the predominant effect of CNQX was on the fast component and, conversely, sodium fluoroacetate primarily inhibited the slow component. Calcium transients were indeed resolved in both astrocytes and neurons following INS using two-photon microscopy. ${ }^{16}$

Calcium dynamics following INS were also investigated in the mouse cerebellum. Previous studies have used flavin autofluorescence imaging, an indirect measure of the rate of mitochondrial ATP production based on the redox state of flavin molecules, such as flavin adenine dinucleotide (FAD) and flavin mononucleotide (FMN), ${ }^{17}$ to assess neuronal activity in the cerebellum. ${ }^{21}$ Due to the neuronal architecture of the cerebellum, which is characterized by long parallel fibers lining the cortical folia, superficial electrical stimulation produces a long-narrow strip of flavin fluorescence and induces field potentials within the strip (beam) of parallel fibers stimulated. Lateral spread (perpendicular to parallel fibers) of the signal is prevented by action of inhibitory interneurons. Previous studies have shown that visualization of the activation along the parallel fibers by flavin imaging requires long electrical stimulation pulse trains (several seconds), presumably related to the time course of mitochondrial activation. However, similarly long pulses of INS do not lead to beam-like activation, but rather to diffuse activation at the stimulation site and is not correlated with the direction of parallel fibers. This diffuse response is eliminated with application of fluoroacetate, an astrocytic poison, suggesting that the activation induced by long train INS is astrocytic in nature. In contrast, stimulation with short INS pulse trains, even just a single optical pulse, results in neural activation, as evidenced by field potential recordings similar to the ones obtained using electrical stimulation. Consistent with this, calcium imaging following INS reveals a fairly focal region of activation, which is detectable at much lower pulse train durations $(\sim 0.2 \mathrm{~s})$ and is insensitive to fluoroacetate. This suggests that INS of short duration, a duration that is too brief to produce detectable changes in flavin fluorescence, activates neuronal response, which is detectable both electrophysiologically and with calcium imaging. Longer INS stimulation leads to accumulation of heat in the tissue, which leads to a delayed but significant astrocytic response. Therefore, the fact that INS can reveal the neural architecture inherent in cerebellar cortex confirms its spatial precision and usefulness as a tool for studying cortical circuitry. Moreover, the markedly different effects of short and long INS pulse trains underscore the importance of choice of stimulation parameters.

\section{INS in Nonhuman Primates}

\subsection{INS Modulates Functional Domain-Specific Sensory Response in Cortex}

INS is attractive for use in nonhuman primate studies, where the same subject is used over the course of months to years and the same area of the brain may be stimulated several hundred times in a single day. The Roe lab at Vanderbilt University is a pioneer of the chronically implanted optical window technique, a method that allows unobscured views of the cortical surface through a biocompatible artificial membrane and adapts it for use in combined imaging and optical stimulation studies. ${ }^{19,22-26}$

Collaborating with the Jansen group, they conducted intrinsic signal imaging of primary visual cortex in response to INS stimulation in anesthetized macaque monkeys [Fig. 1(a)]. They found that INS stimulation produced responses typical of visually induced cortical intrinsic signals. These responses were intensity and duration dependent [Fig. 1(b)] and induced robust increases in neuronal activity immediately following optical stimulation [Fig. 1(c)]. They further demonstrated effects of INS stimulation that were specific to stimulated functional domains. In monkey primary visual cortex, visual information from each eye is represented in eye-specific domains called ocular dominance columns [Fig. 1(d): L, left eye column; $\mathrm{R}$, right eye column]. To examine whether INS could be used to modulate activity in functionally specific domains, Cayce et al. ${ }^{7}$ targeted INS to stimulate individual ocular dominance columns; eye-specific INS stimulation was achieved with small diameter optical fibers (100 to 200 micron core diameters), which produced spot sizes confined to single ocular dominance columns [Fig. 1(d): yellow dot]. The results of such INS stimulation demonstrated functionally specific modulation of visual stimulation. Specifically, INS stimulation of a left eye column led to enhancement of imaged response to visual stimulation of the left eye [Fig. 1(e): increased reflectance change from baseline, green curve to black curve] and relative suppression of signals from the right eye columns [Fig. 1(f): reduction in reflectance change from baseline, green curve to black curve]. [Note that in intrinsic signal imaging, a larger negative reflectance change, $\mathrm{dR} / \mathrm{R}$, indicates an increased response and a smaller negative reflectance change indicates a reduced response].

Interestingly, by comparing 100-, 200-, and 400- $\mu$ m-diameter fibers, Cayce et al. found that stimulation spot size matters [Fig. 1(g)]. When INS stimulation alone was used, the larger the fiber diameter, the larger the induced response [Fig. 1(h): blue to red to green], consistent with recruitment of a greater population of neurons. However, when used in combination with visual stimulation, the cortex exhibited behavior dependent on the functional organization of the cortex. That is, addition of INS stimulation to visual stimulation with the smaller fibers (100 or $200 \mu \mathrm{m}$ ) led to some increase in response size, as expected [Fig. 1(i): compare blue in (h) and (i), and red in (h) and (i)]. However, application of INS with the large $(400 \mu \mathrm{m})$ fiber led to a drastic reduction in response [Fig. 1(i): compare green in (h) and (i)]. This result was likely due to the spot size exceeding the extent of the single eye column (single eye columns typically $400 \mu \mathrm{m}$ in width) and impinging the other eye column. Since 
INS Has Excitatory Effect on Neurons
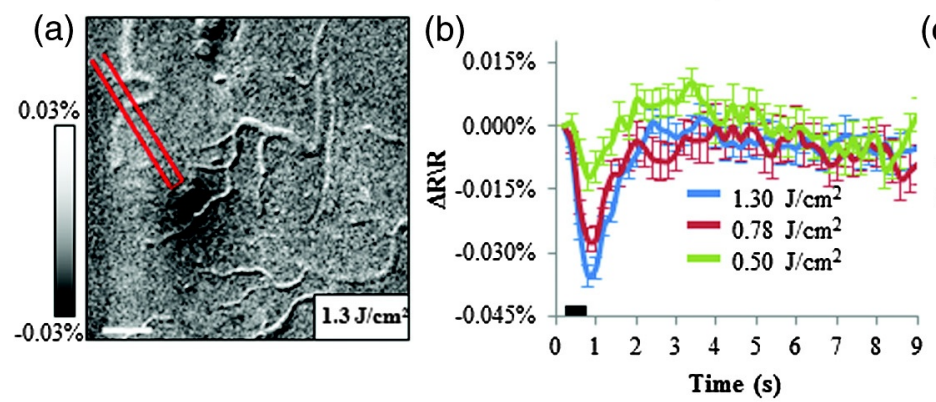

Focal Stimulation Elicits Functional Domain Specific Response

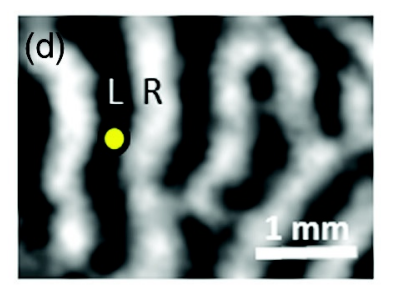

Stimulation in Left Eye Column Elicits Responses in $E$ and $F$

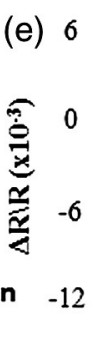

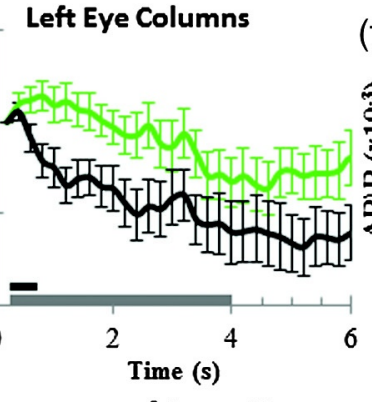

Importance of Spot Size (g)

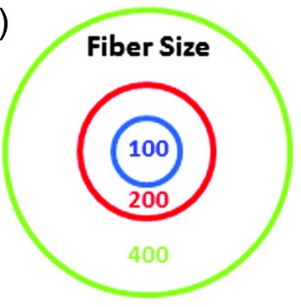

(h)

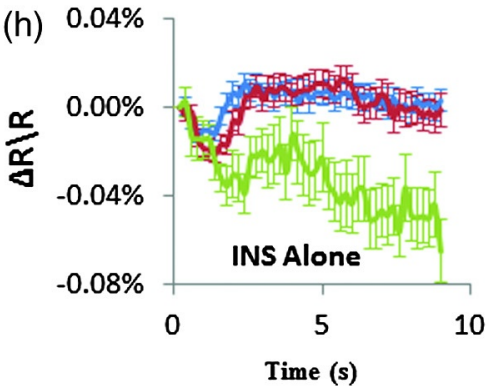

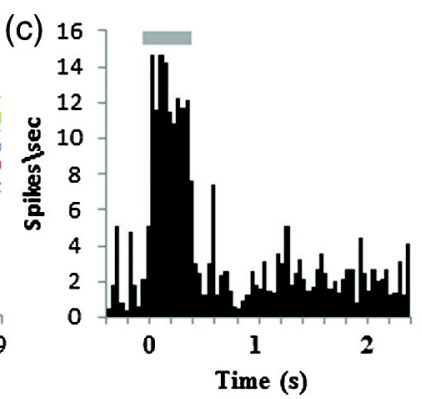
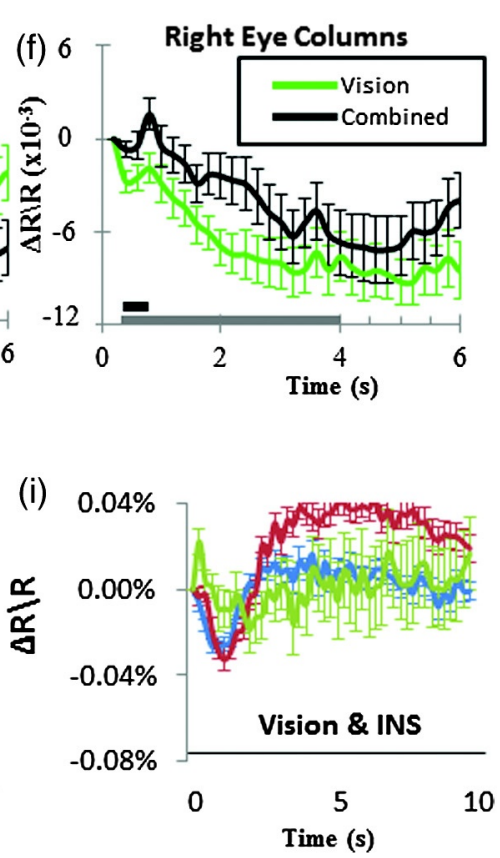

Fig. 1 Effect of infrared neural stimulation (INS) on cortical response in macaque monkeys. (a) to (c) INS induces excitatory neuronal response. (a) Optical image of INS stimulation in monkey visual cortex. Red outline: 100-um optical fiber. Radiant exposure: $1.3 \mathrm{~J} / \mathrm{cm}^{2}$. (b) Increasing radiant exposure increases magnitude of response. Timecourse of imaged reflectance signal in response to three levels of radiant exposure per pulse. Black bar: period of INS. (c) Neural response to INS stimulation (gray bar). INS stimulation parameters [(a) to (c): $\lambda=1.875 \mu \mathrm{m}$, pulse width $=250 \mu \mathrm{s}$, pulse train length $=500 \mathrm{~ms}$, fiber size $=100 \mu \mathrm{m}$. Imaging parameters: $5 \mathrm{fps}$, inter-trial inteval $=8 \mathrm{~s}$, number of trials $=40$ $\left(1.3 \mathrm{~J} / \mathrm{cm}^{2}\right), 22\left(0.78 \mathrm{~J} / \mathrm{cm}^{2}\right)$, and $20\left(0.50 \mathrm{~J} / \mathrm{cm}^{2}\right)$. Scale bar in (a): $1 \mathrm{~mm}$. A, anterior; L, lateral. (d) to (f) INS elicits functional domain specific response. (d) Ocular dominance map from macaque primary visual cortex. Yellow dot: location of $200-\mu \mathrm{m}$ optical fiber in a left eye ocular dominance column. INS stimulation of left eye column enhances effect of visual stimulation in left eye column (e) and reduces effect in right eye column (f). Black: in response to visual + INS. Green: reflectance signal in response to visual stimulation alone. Black bars in (e) and (f) represent INS, and gray bars represent visual stimulation. (g) and (h) Effect of optical fiber diameter. (g) Blue: $100 \mu \mathrm{m}$. Red: $200 \mu \mathrm{m}$. Green: $400 \mu \mathrm{m}$. (h) Larger INS spot size produces larger cortical response. (i) Combining INS and visual stimulation enhances the response when spot size is confined to single ocular dominance column (blue, red). However, INS dramatically reduces the visual response when spot size invades other eye column (green). The onset time and duration of visual stimuli and INS are the same as in (e) and (f). Adapted from Ref. 7.

the eye columns have push-pull effects on one another, activation of the opposing eye column led to significant reduction of left eye column response. These findings are quite exciting as they suggest that this method could be used to modulate specific functionalities related to known functional organization in the brain. Importantly, it cautions against the use of stimulation without a detailed knowledge of underlying functional organization and underscores the need for high spatial resolution stimulation methods.

\subsection{INS as a Functional Tract Tracing Methodology}

The potential use of INS as a tool for functional tract tracing was also investigated. Previous studies have demonstrated the use of focal electrical stimulation with optical imaging to map intra-areal or inter-areal connections. ${ }^{27,28}$ However, the mapping of more global connections is better conducted with functional MRI (fMRI) methods, an approach that lacks the spatial resolution of intrinsic optical imaging but has the 
important advantage of being able to resolve changes in subsurface structures. Indeed, the potential for using light-based methods with fMRI for functional tract tracing has already been demonstrated. ${ }^{29-31}$

Recently, Chen et al. were able to image INS-induced activation of the somatosensory cortex in a high field 9.4T scanner. INS stimulation was presented through an MRI-optimized optical window implanted in a squirrel monkey [Fig. 2(a)], while imaged slices were obtained in the tangential [Fig. 2(b), red] or sagittal [Fig. 2(b), green] planes. ${ }^{9}$ Slices imaged in the tangential plane revealed that optical stimulation of the somatotopic representation of a single digit in area 1 of the monkey somatosensory cortex produced focal blood-oxygen-level dependent (BOLD) signals not only at the stimulation site [Fig. 2(a), yellow dot] but also within topographically matching areas $3 \mathrm{a}, 3 \mathrm{~b}$, and 2 [Fig. 2(a), white dots] of somatosensory cortex. Slices imaged in the sagittal plane revealed lamina-specific activations in target areas; specifically, the middle layers in areas $3 \mathrm{a}$ and $3 \mathrm{~b}$ and the superficial layers in area 2, consistent with known feedback and feed forward cortico-cortical connection patterns. An additional interesting finding of the study was that although INS acts primarily on the superficial layers, at the site of stimulation, BOLD signal was detected throughout the cortical layers, including the deeper cortical layers. This suggests that INS triggers additional effects in the neuronal network that may or may not be detected using optical imaging methods, which derive their signal from the superficial layers.

\subsection{INS as a Behavioral Modulator}

Although the efficacy of INS has been demonstrated using a variety of techniques (electrophysiology, optical imaging, fMRI), the picture would not be complete without evidence of a behavioral effect of INS in awake animals. Whether this could be done or not was difficult to predict. Although the spatial specificity is considered a strong advantage of INS, it was unknown whether such focal activation could activate sufficient neuronal circuitry to trigger changes in behavior. Indeed, behavioral effects of optogenetic techniques in nonhuman primates have been notoriously difficult to observe and have generally revealed only a small statistical bias of one mode of behavior over another in a course of hundreds of trials. ${ }^{31}$ This is generally believed to be due to the sparse expression of photosensitive neurons in the brain and a reliable but not dramatic increase in their firing rates following optical stimulation.

A recent study examined the potential for INS to induce eye movement behavior in an awake monkey. ${ }^{32}$ A macaque monkey implanted with an optical chamber over the visual cortex was trained to sit quietly in front of a computer monitor. During each trial, it was asked to fixate with its eyes on a small dot. Afterward, the monkey was rewarded with a drop of water and then allowed to look wherever it pleased until the start of the next trial. The direction of the animal's gaze was constantly recorded using an infrared eye tracker. By mapping (in a previous session) the visuotopy represented within the chamber [Fig. 2(d)], the investigators predicted that focal INS stimulation at a specific cortical location [Figs. 2(d) and 2(e): yellow dot] would produce a phosphene (perception of a small spot of light) at a specific location in visual space. ${ }^{33}$ As monkeys naturally look at targets that appear, the expectation is that the monkey would look at (saccade toward) the predicted visual location. In most trials, INS was not delivered, and the monkey would remain fixated on the dot, receive its reward, and break off its gaze in a random trajectory. In a fraction of the trials, which were randomly chosen, INS was delivered a brief period after the animal was given its reward. In these trials, the monkey would consistently look at the visual location corresponding to the retinotopic representation at the stimulated cortical site [Fig. 2(f): blue arrow]. This result suggests that INS induced
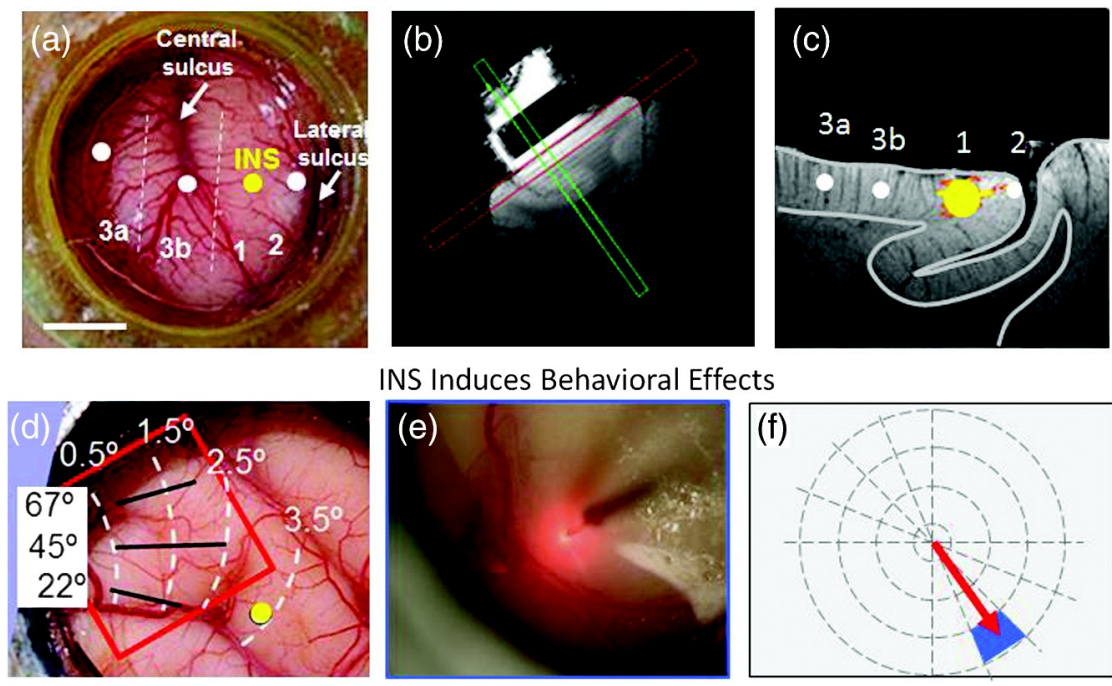

INS Induces Behavioral Effects
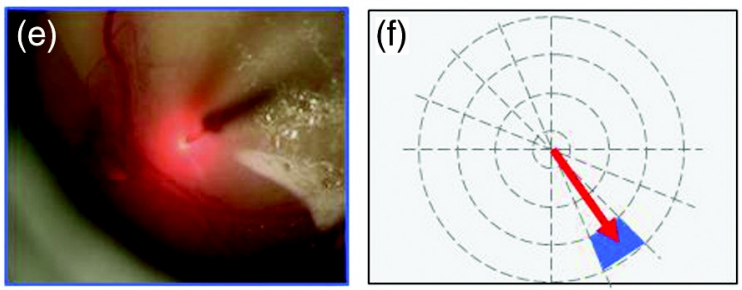

Fig. 2 Other applications of INS in central nervous system. (a) to (c) Functional tract tracing tool. (a) View of somatosensory cortex (areas 3a, 3b, 1, and 2) seen through magnetic resonance imaging (MRI)-compatible optical chamber in squirrel monkey. Dots: locations of significant blood-oxygen-level dependent (BOLD) signal obtained in response to INS stimulation at yellow dot. (b) Structure MRI with tangential (red) and sagittal (green) slices indicated. (c) Sagittal slice with locations of significant BOLD activations in response to INS stimulation in area 1 (yellow dot). Activations are confined to specific cortical laminae. (d) to (f) Induction of behavioral effect. (d) Visuotopic mapping of V4 in macaque monkey (cf. Ref. 23). White dotted lines: isoeccentricities. Black solid lines: isopolarities. Yellow dot: INS fiber optic location. (e) Fiber optic applied to visual cortex. (f) INS at specific cortical site leads to eye movements to visuotopic location represented at that site. 
perceptions that led to predicted behaviors and is the first demonstration of the ability of INS to influence behavior.

\section{Future of INS}

The first papers on INS and optogenetics were both published around 2005. Almost a decade has passed and optogenetics has revolutionized neuroscience. The technology has been developed on a large scale, with viral opsin vectors being available from several core facilities and transgenic mouse models being marketed by several commercial vendors. INS, on the other hand, has remained relatively obscure, confined only to a handful of laboratories. We think that there are several reasons why INS failed to gain momentum. Optogenetics, innovative as it was, was largely a successful exercise in molecular biology. And since molecular biology is a basic part of neuroscience training, it could be readily understood and accepted by neuroscientists. In short, the people who developed the technique and the ones that used it spoke the same language. The light sources used for optogenetics were also fairly commonplace in light microscopy. INS, in 2005, on the other hand, required large expensive liquidcooled lasers. These were either clinical systems designed for tissue ablation or devices for physics research. Both required special training to operate. The thermal nature of INS led critics to dismiss it as controlled destruction of tissue rather than anything else, and before 2012, no satisfactory theories of how heat delivery leads to action potentials existed. Today, however, the situation has changed dramatically. First, the light sources necessary for INS are more readily accessible and less daunting. High-power (several hundred milliwatts) infrared diodes are available from several manufacturers and can be efficiently coupled to optical fibers in a setup very similar to the one used in optogenetics. The defocusing effect of the fiber also effectively limits their range of action to within a few millimeters, alleviating most safety concerns for the operator. The safety of INS has been demonstrated both in the peripheral and in the central nervous system, including the nonhuman primate brain. ${ }^{34-36}$ Safe stimulation parameters have been published and if one is interested in experimenting with other stimulation sequences, very good computer models for estimating the thermal changes of INS are now available. ${ }^{37}$ Theoretical modeling and experimental results show that INS requires a change of temperature of the order of a few degrees (and a fraction of a degree in some tissues), values comparable to the temperature changes observed during optogenetic stimulation. ${ }^{3}$

We conclude that although INS has been slow in gaining momentum, its outlook as a tool for neuroscience is bright (no pun intended!). The technological obstacles for using INS as a neural stimulation method have been largely overcome and its safety and efficacy have been demonstrated. It offers several unique advantages over other technologies. First, it allows for very focal stimulation, which is difficult to achieve with electrical stimulation. Second, it can be used to stimulate neural tissue without physical contact. Third, in contrast to multiple insertions of electrodes or optrodes, stimulation at different sites is relatively easy, as one simply moves the illumination spot from one location to another. Fourth, much emphasis is placed today on multimodal techniques for investigating brain function. INS does not interfere with electrical recordings (unlike electrical stimulation) and is compatible with optical imaging techniques and MRI. Finally, unlike optogenetics, it does not require genetic manipulation, which has proven difficult in nonhuman primates and is not available for use in humans. Given its unique set of advantages, we believe that INS will soon gain its proper place as a scientific and clinical tool.

\section{Acknowledgments}

The authors would like to acknowledge financial support from the National Institutes of Health through grants NS044375 and MH095009 to A.W.R.

\section{References}

1. V. S. Polikov, P. A. Tresco, and W. M. Reichert, "Response of brain tissue to chronically implanted neural electrodes," J. Neurosci. Methods 148(1), 1-18 (2005).

2. E. S. Boyden, "A history of optogenetics: the development of tools for controlling brain circuits with light," Biol. Rep. 3, 11 (2011).

3. O. Yizhar et al., "Optogenetics in neural systems," Neuron 71(1), 9-34 (2011).

4. J. Wells et al., "Optical stimulation of neural tissue in vivo," Opt. Lett. 30(5), 504-506 (2005).

5. J. Wells et al., "Biophysical mechanisms of transient optical stimulation of peripheral nerve," Biophys. J. 93(7), 2567-2580 (2007).

6. M. G. Shapiro et al., "Infrared light excites cells by changing their electrical capacitance," Nat Commun. 3, 736 (2012).

7. J. M. Cayce et al., "Infrared neural stimulation of primary visual cortex in non-human primates," Neuroimage 84, 181-190 (2014).

8. J. Wells et al., "Application of infrared light for in vivo neural stimulation," J. Biomed. Opt. 10(6), 064003 (2005).

9. G. Chen et al., Eds., "Functional tract tracing in non-human primates using pulsed infrared lasers in conjunction with optical imaging and fMRI," presented at Society for Neuroscience Meeting, Society for Neuroscience, New Orleans, LA (2012).

10. S. L. Jacques, "Laser-tissue interactions. Photochemical, photothermal, and photomechanical," Surg. Clin. North Am. 72(3), 531-558 (1992).

11. J. M. Cayce et al., "Infrared neural stimulation of thalamocortical brain slices," IEEE J. Sel. Topics Quantum Electron. 16(3), 565-572 (2010).

12. E. S. Albert et al., "TRPV4 channels mediate the infrared laser-evoked response in sensory neurons," J. Neurophysiol. 107(12), 3227-3234 (2012).

13. S. M. Rajguru et al., "Optical cochlear implants: evaluation of surgical approach and laser parameters in cats," Hear. Res. 269(1-2), 102-111 (2010).

14. A. D. Izzo et al., "Selectivity of neural stimulation in the auditory system: a comparison of optic and electric stimuli," J. Biomed. Opt. 12(2), 021008 (2007).

15. M. W. Jenkins et al., "Optical pacing of the embryonic heart," Nat. Photonics 4(9), 623-626 (2010).

16. J. M. Cayce et al., "Calcium imaging of infrared-stimulated activity in rodent brain," Cell Calcium 55(4), 183-190 (2014).

17. M. M. Chernov et al., Eds., "Infrared neuronal stimulation of the cerebellum: developing a versatile tool for modulating neuronal activity," presented at Society for Neuronscience Meeting, Society for Neuroscience, Washington, DC (2011).

18. A. Grinvald et al., "Optical imaging of neuronal activity," Physiol. Rev. 68(4), 1285-1366 (1988).

19. A. W. Roe, "Long-term optical imaging of intrinsic signals in anesthetized and awake monkeys," Appl. Opt. 46(10), 1872-1880 (2007).

20. J. M. Cayce et al., "Pulsed infrared light alters neural activity in rat somatosensory cortex in vivo," Neuroimage 57(1), 155-166 (2011).

21. K. C. Reinert et al., "Cellular and metabolic origins of flavoprotein autofluorescence in the cerebellar cortex in vivo," Cerebellum 10(3), 585599 (2011).

22. H. D. Lu et al., "A motion direction map in macaque V2," Neuron 68(5), 1002-1013 (2010).

23. H. Tanigawa, H. D. Lu, and A. W. Roe, "Functional organization for color and orientation in macaque V4," Nat. Neurosci. 13(12), 15421548 (2010).

24. O. Ruiz et al., "Optogenetics through windows on the brain in the nonhuman primate," J. Neurophysiol. 110(6), 1455-1467 (2013).

25. G. Chen et al., Eds., "Functional tract tracing in non-human primates using pulsed infrared lasers in conjuction with optical imaging and fMRI," presented at Society for Neuroscience Meeting, New Orleans, LA (2012). 
26. A. W. Roe et al., Eds., "Infrared neural stimulation of visual cortex induces visual perception in awake non-human primates," presented at Society for Neuroscience Meeting, San Diego, CA (2013).

27. A. A. Brock et al., "Optical imaging of cortical networks via intracortical microstimulation," J. Neurophysiol. 110(11), 2670-2678 (2013).

28. J. H. Kaas, O. A. Gharbawie, and I. Stepniewska, "Cortical networks for ethologically relevant behaviors in primates," Am. J. Primatol. 75(5), 407-414 (2013)

29. M. Desai et al., "Mapping brain networks in awake mice using combined optical neural control and fMRI," J. Neurophysiol. 105(3), 1393-1405 (2011).

30. I. Kahn et al., "Characterization of the functional MRI response temporal linearity via optical control of neocortical pyramidal neurons," J. Neurosci. 31(42), 15086-15091 (2011).

31. A. Gerits et al., "Optogenetically induced behavioral and functional network changes in primates," Curr. Biol. 22(18), 1722-1726 (2012).

32. G. Chen et al., Eds., "Optical control of the visual perception of awake non-human primate with infrared neural stimulation," presented at SPIE Photonics West, SPIE, San Francisco, CA (2013).

33. E. M. Schmidt et al., "Feasibility of a visual prosthesis for the blind based on intracortical microstimulation of the visual cortex," Brain 119(Pt 2), 507-522 (1996).

34. J. D. Wells et al., "Optically mediated nerve stimulation: identification of injury thresholds," Lasers Surg. Med. 39(6), 513-526 (2007).
35. V. Goyal et al., "Acute damage threshold for infrared neural stimulation of the cochlea: functional and histological evaluation," Anat. Rec. (Hoboken) 295(11), 1987-1999 (2012).

36. M. M. Chernov, G. Chen, and A. W. Roe, "Histological assessment of thermal damage in the brain following infrared neural stimulation," Brain Stimul. 7(3), 476-482 (2014).

37. A. Thompson et al., "Infrared neural stimulation: influence of stimulation site spacing and repetition rates on heating," IEEE Trans. Biomed. Eng. 60(12), 3534-3541 (2013).

Mykyta Chernov is a postdoctoral research scholar at the Department of Psychology at Vanderbilt University. He received his $\mathrm{MS}$ in biomedical engineering and his $\mathrm{PhD}$ in physiology from Dartmouth College. He is interested in developing novel engineering and computational techniques to address unanswered questions in neuroscience.

Anna Wang Roe is a professor of psychology, radiology and biomedical engineering at Vanderbilt University. She received her BS from Harvard University and her $\mathrm{PhD}$ in neuroscience from the Massachusetts Institute of Technology. She studies the functional organization of cortical sensory areas with an emphasis on imaging methods. 